\title{
A state-of-charge estimation method of the power lithium-ion battery in complex conditions based on adaptive square root extended Kalman filter.
}

JIANG, C., WANG, S., WU, B., FERNANDEZ, C., XIONG, X. and COFFIEKEN, J. 


\title{
A state-of-charge estimation method of the power lithium-ion battery in complex conditions
}

\section{based on adaptive square root extended Kalman filter}

\author{
Cong Jiang ${ }^{\mathrm{a}}$, Shunli Wang ${ }^{\mathrm{a}}$, Bin Wu ${ }^{\mathrm{a}}$, Carlos Fernandez ${ }^{\mathrm{b}}$, Xin Xiong ${ }^{\mathrm{a}}$, James Coffie-Ken $^{\mathrm{a}}$ \\ ${ }^{a}$ School of Information Engineering, Southwest University of Science and Technology, Mianyang, 621010, China \\ ${ }^{\mathrm{b}}$ School of Pharmacy and Life Sciences, Robert Gordon University, Aberdeen, AB10-7GJ, UK
}

\begin{abstract}
The control strategy of electric vehicles mainly depends on the power battery state-of-charge estimation. One of the most important issues is the power lithium-ion battery state-of-charge (SOC) estimation. Compare with the extended Kalman filter algorithm, this paper proposed a novel adaptive square root extended Kalman filter together with the Thevenin equivalent circuit model which can solve the problem of filtering divergence caused by computer rounding errors. It uses Sage-Husa adaptive filter to update the noise variable, and performs square root decomposition on the covariance matrix to ensure its non-negative definiteness. Moreover, a multi-scale dual Kalman filter algorithm is used for joint estimation of SOC and capacity; the forgetting factor recursive least-square method is used for parameter identification. To verify the feasibility of the algorithm under complicated operating conditions, different types of dynamic working conditions are performed on the ternary lithium-ion battery. The proposed algorithm has robust and accurate SOC estimation results and can eliminate computer rounding errors to improve adaptability compared to the conventional extended Kalman filter algorithm.
\end{abstract}

Keywords: state-of-charge; extended Kalman filter; adaptive; square root; power lithium-ion battery

*Corresponding author: Shun-Li Wang. Tel: +86-15884655563. E-mail address: 497420789@qq.com.

1. Introduction

In order to deal with environmental problems, clean energy related research has become a hot spot in recent years. The lithium-ion battery (LIB) is becoming increasingly popular[1, 2]. LIB plays an important role in power supply applications such as electric vehicles (EV), underwater vehicles, and distributed energy storage system [3]. The safety of the LIB is still an issue, which affects the utilization efficiency of its capacity and life directly, leading to accidents in several cases [4]. To ensure safe and reliable operation, LIB needs to be monitored and managed by the Battery Management Systems (BMS) [5]. The accurate SOC estimation by the BMS is necessary for the power application LIB[6]. However, the technical bottleneck of the accurate SOC estimation is an insurmountable problem [7], due to the immature management of the associated BMS equipment. It is used for the energy supply, which has stability and reliability requirements [8], aiming to avoid safety issues by monitoring battery status. 
Many researchers have studied the LIB and proposed many methods to estimate the SOC until now. Most of existing SOC estimation methods can be divided into the following categories: (1) direct measurement method; (2) data-driven estimation method; (3) model-based estimation method [9, 10].

(1) Direct measurement method: The direct measurement method mainly includes the coulomb counting method and open-circuit voltage (OCV) method. The SOC value can be obtained by integrating the current when the initial value of the SOC is known. This method is called the coulomb counting method or ampere-hour integral method which is an open-loop method. It is sensitive to the initial value of SOC, and will cause uncorrectable cumulative errors. The relationship between OCV and SOC can be obtained by a fixed discharge rate, and then the value of the corresponding SOC is found in the relationship curve using the known OCV [11]. This method is called the OCV method [12]. Although the method can measure the value of SOC [13], the battery must be allowed to stand for more than one hour to start measuring, and the battery itself is susceptible to temperature and reproductive quality $[14,15]$. Therefore, it is not suitable for SOC estimation in operation.

(2) Data-driven estimation method: Data-driven estimation methods use the input-output data of the system to develop an estimator. Since these methods do not require an accurate plant model, the estimations and assumptions introduced in the plant modeling step are omitted[9]. The typical algorithms include the fuzzy controller, the neural network[16-19], and the support vector machine(SVM)[20]. But data-driven estimation method suffers from problems like extensive training, difficult online adaption, and high computational effort[21].

(3) Model-based estimation method: With the development of battery research, many battery models for the power LIB have been proposed[22-26]. Commonly used models can be roughly summarized into three types: electrochemical models (EM), equivalent circuit models (ECM), and electrochemical impedance models (EIM). In the model-based estimation method, the battery model is generally expressed as state equations. Several state observers have been applied to the models separately, including extended Kalman filter (EKF), unscented Kalman filter (UKF) sliding mode observer (SMO), particle filter (PF), H-infinity observer, and their improved algorithms [10, 18, 27-34].

The battery characteristics are obtained by performing capacity and hybrid pulse power characteristic (HPPC) experiments on the battery at different temperatures, and analyzing the open-circuit voltage, ohmic internal resistance, and polarization resistance of the LIB under different states of charge and temperature. The parameters identification of the LIB model is carried out by the forgetting factor recursive least square (FFRLS) method. Co-estimate capacity and SOC based on a multiscale framework with EKF. Because all algorithm programs run on the computer, the realization of the Kalman filter maybe have numerical problems which can be proved by some papers [35, 36]. There may be calculation errors due to the limited word length of the computer. When the error accumulation is serious, the error variance matrix $P_{\mathrm{k}}$ can gradually lose its positive definiteness or symmetry, leading to Kalman filter divergence. To 
solve this problem, the square root decomposition is used to improve the EKF algorithm for the estimation of SOC. The Kalman algorithm and the EKF algorithm both treat system noise like white noise and ignore the noise characteristics in practical applications, thereby leading to the noise affecting the accuracy of the SOC estimation. An extended Kalman filter method based on adaptive covariance matching algorithm is used in some paper [34]. The noise is adjusted by Sage-Husa adaptive filtering and the adaptive square root extended Kalman filter (ASREKF) is a combined algorithm in this paper.

The rest of this paper is organized as follows. The mathematical theoretical analysis is conducted in section 2 including a definition of SOC, modeling of the LIB, and proposed ASREKF algorithm. In section 3, the experiments are illustrated as well as their estimation results. The conclusions is finally reported in Section 4.

\begin{tabular}{|llll|}
\hline \multicolumn{2}{ll}{ Nomenclature } & & \\
SOC & state-of-charge & EV & Electric vehicle \\
LIB & lithium-ion battery & ECM & Equivalent circuit model \\
KF & Kalman filter & EM & Electrochemical model \\
FFRLS & forgetting factor recursive least-square & EIM & Electrochemical impedance model \\
PF & Particle filter & RC & Resistor-capacitor \\
EKF & Extended Kalman filter & UKF & Unscented Kalman filter \\
HPPC & Hybrid Pulse Power Characteristic & ANN & Artificial neural network \\
ASREKF & Adaptive square root extended Kalman filter & CC-CV & Constant current-constant voltage \\
BBDST & Beijing Bus Dynamic Stress Test & OCV & Open circuit voltage \\
MAE & Mean Absolute Error & RSME & Root mean square error \\
DOD & depth of discharge & SVM & support vector machine \\
\hline
\end{tabular}

2. Mathematical analysis

\subsection{Definition of SOC}

The SOC characterizes the remaining capacity of LIB, and is defined as the ratio of the remaining capacity to the maximum available capacity which can be expressed as Eq. (1)[10].

$$
\left\{\begin{array}{l}
S O C_{t}=1-D O D_{t}=\frac{C_{t}}{C_{\max }} \times 100 \% \\
S O C_{t}=S O C_{0}-\frac{\int_{t_{0}}^{t} \eta i(t) \eta d t}{C_{\max }}
\end{array}\right.
$$

In Eq. (1), $S O C_{t}$ is the current estimated $\mathrm{SOC}, D O D_{t}$ is the depth of discharge, $C_{t}$ is the remaining battery capacity, $C_{\max }$ is the maximum available capacity when the battery is fully charged, $S O C_{0}$ is the initial $\mathrm{SOC}$ when the estimation process starts, $\eta$ denotes the Coulombic efficiency, and $i(t)$ is the load current (assumed discharging is positive).

\subsection{Modeling of $L I B$}

At present, there are some equivalent models for LIB which are proposed. Since the equivalent circuit model has the advantages of simple calculation and clear physical meaning, it is the most widely used. The simple model is easy to 
calculate, but it can not accurately describe the working characteristics of the battery. The complex model can better characterize the charge and discharge characteristics of the battery, but the calculation amount is greatly increased. Therefore, it is necessary to comprehensively consider accurate modeling and model simplicity. Many researchers choose the Thevenin equivalent circuit model $[21,34,37,38]$ or second-order equivalent circuit model $[39,40]$ after considering the model complexity and accuracy. Lai compared the influence of different order equivalent circuit models on the SOC estimation effect [41]. The results show that in the use of the second-order equivalent circuit, the accuracy of the model does not increase significantly, but the number of calculations based on the number of parameters increases. Considering the needs of engineering applications, the dynamic characteristics of LIB should be characterized by an uncomplicated equivalent model, so the Thevenin equivalent circuit model is selected. This paper uses the Thevenin equivalent circuit model shown in Fig.1.

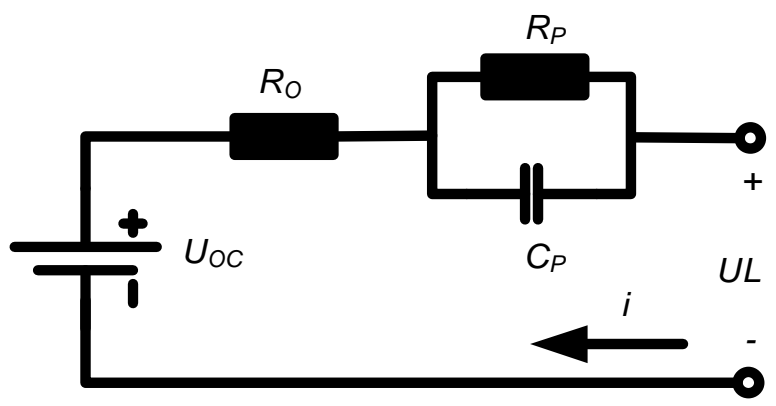

Fig. 1 Thevenin equivalent circuit model

In Fig.1, $U_{o c}$ represents the $\mathrm{OCV}$ of the $\mathrm{LIB}, R_{O}$ represents the ohmic internal resistance, $R_{P}$ represents the polarization resistance of the lithium battery, $C_{P}$ represents the polarization capacitance, and the RC network characterizes the polarization phenomenon of the LIB, $i$ indicates the load current, and $U_{L}$ indicates the closed-circuit voltage when the battery pack is externally connected. The circuit equation can be listed according to the Kirchhoff laws as follows:

$$
\begin{gathered}
U_{L}=U_{O C}-R_{o} i-U_{p} \\
\frac{d U_{p}}{d t}=\frac{i}{C_{p}}-\frac{U_{p}}{C_{p} R_{p}}
\end{gathered}
$$

$U_{p}$ represents the polarization voltage. Eq. (3) can be rewritten in the discrete-time form as[34, 42]:

$$
U_{p, k}=U_{p, k-1} \exp (-\Delta t / \tau)+R_{P}(1-\exp (-\Delta t / \tau)) i_{k-1}
$$

In Eq. (4), $\Delta t$ is a fixed time step between two adjacent measurement points and $\tau=R_{p} C_{p}$.

$$
\begin{aligned}
& U_{L, k}-U_{O C, k}=-U_{p, k}-R_{O} i_{k}=-U_{p, k-1} \exp (-\Delta t / \tau)-R_{P}(1-\exp (-\Delta t / \tau)) i_{k-1}-R_{O} i_{k} \\
& =\exp (-\Delta t / \tau)\left(U_{L, k-1}-U_{O C, k-1}\right)-R_{O} i_{k}+\left(\exp (-\Delta t / \tau) R_{O}-R_{P}(1-\exp (-\Delta t / \tau))\right) i_{k-1}
\end{aligned}
$$

Then, define $U_{d, k}=U_{L, k}-U_{O C, k}$, we can get: 


$$
U_{d, k}=\alpha_{0} U_{d, k-1}+\alpha_{1} i_{k}+\alpha_{2} i_{k-1}
$$

In Eq. (6), the model coefficient $\alpha_{0}, \alpha_{1}$ and $\alpha_{2}$ are:

$$
\left\{\begin{array}{l}
\alpha_{0}=\exp (-\Delta t / \tau) \\
\alpha_{1}=-R_{O} \\
\alpha_{2}=\left(\exp (-\Delta t / \tau) R_{O}-R_{P}(1-\exp (-\Delta t / \tau))\right)
\end{array}\right.
$$

The open-circuit-voltage $U_{o c}$ can be characterized by the state variable SOC, according to which the nonlinear function relationship can be obtained. The function of $U_{o c}$ and SOC can be expressed as Eq. (8).

$$
U_{O C, k}=G\left(S O C_{k}\right)=k_{0}+k_{1} S O C_{k}+k_{2} S O C_{k}^{2}+k_{3} S O C_{k}^{3}+k_{4} S O C_{k}^{4}+k_{5} S O C_{k}^{5}
$$

\subsection{Model parameters online identification}

The $R_{O}, C_{p}$, and $R_{p}$ of LIB model parameters vary with temperature and SOC, which can be seen in section 3.3. Model parameters may be changed by internal or external factors (such as battery aging or changes in the operating environment), and it can not be tracked and corrected in real-time by using conventional offline identification methods. To solve this problem, the online parameter identification method based on the forgetting factor RLS method is used in the model parameter identification [34].

Assume the model can be described as:

$$
y_{k}=\varphi_{k} \theta_{k}+\xi_{k}
$$

Then the system identification can be realized as follows:

$$
\left\{\begin{array}{l}
L_{k}=\frac{P_{k-1} \varphi_{k}^{T}}{\lambda+\varphi_{k} P_{k-1} \varphi_{k}^{T}} \\
P_{k}=\lambda^{-1}\left(P_{k-1}-L_{k} \varphi_{k} P_{k-1}\right) \\
e_{k}=y_{k}-\varphi_{k} \hat{\theta}_{k-1} \\
\hat{\theta}_{k}=\hat{\theta}_{k-1}+L_{k} e_{k}
\end{array}\right.
$$

In Eq. (8) $\lambda$ is the forgetting factor and $e_{k}$ is the prediction error. The model can be estimated iteratively by the recursive equations of the EKF, which are given in Eq. (10). The detailed implementation process of the forgetting factor RLS algorithm is shown in table 1.

Table 1 Summary of the forgetting factor RLS algorithm.

1) Parameter identification of OCV model in Eq. (3)

2) Parameter identification of battery model in Eq. (7)

a) Initialization:

a) Initialization:

$$
\begin{aligned}
& \varphi_{1, k \mid k=0}=\left[\begin{array}{llllllll}
1 & S O C_{0} & S O C_{0}^{2} & S O C_{0}^{3} & S O C_{0}^{4} & S O C_{0}^{5}
\end{array}\right] \quad \varphi_{2, k \mid k=0}=\left[\begin{array}{llll}
\left(U_{L, 1}-U_{O C, 1}\right) & i_{1} & i_{0}
\end{array}\right] \\
& \theta_{1, k \mid k=0}=\left[\begin{array}{llllll}
k_{0} & k_{1} & k_{2} & k_{3} & k_{4} & k_{5}
\end{array}\right] \quad \theta_{2, k \mid k=0}=\left[\begin{array}{lll}
\alpha_{0} & \alpha_{1} & \alpha_{2}
\end{array}\right] \\
& P_{1, k \mid k=0}=\delta_{1} I\left(\delta_{1}=10^{6}\right), \lambda_{1} \in[0.95,1] \\
& P_{2, k \mid k=0}=\delta_{2} I\left(\delta_{2}=10^{6}\right), \lambda_{2} \in[0.95,1]
\end{aligned}
$$


b) Calculate algorithm gain $L_{1, k}$ and error covariance b) Calculate algorithm gain $L_{2, k}$ and error covariance matrix $P_{1, k}$ : matrix $P_{2, k}$ :

$L_{1, k}=\frac{P_{1, k-1} \varphi_{1, k}^{T}}{\lambda_{1}+\varphi_{1, k} P_{1, k-1} \varphi_{1, k}^{T}}, P_{1, k}=\lambda_{1}^{-1}\left(P_{1, k-1}-L_{1, k} \varphi_{1, k} P_{1, k-1}\right) \quad L_{2, k}=\frac{P_{2, k-1} \varphi_{2, k}^{T}}{\lambda_{2}+\varphi_{2, k} P_{2, k-1} \varphi_{2, k}^{T}}, P_{2, k}=\lambda_{2}^{-1}\left(P_{2, k-1}-L_{2, k} \varphi_{2, k} P_{2, k-1}\right)$

c) Calculate prediction error $e_{1, k}$ and update model c) Calculate prediction error $e_{2, k}$ and update model parameters: parameters:

$e_{1, k}=y_{1, k}-\varphi_{1, k} \hat{\theta}_{1, k-1}, \hat{\theta}_{1, k}=\hat{\theta}_{1, k-1}+L_{1, k} e_{1, k} \quad e_{2, k}=y_{2, k}-\varphi_{2, k} \hat{\theta}_{2, k-1}, \hat{\theta}_{2, k}=\hat{\theta}_{2, k-1}+L_{2, k} e_{2, k}$

\subsection{Adaptive estimation algorithm}

\subsubsection{A multiscale framework with extended Kalman filter}

Compared with the change of the SOC, the battery capacity changes very slowly over time. Therefore, we will use a discrete-time state-space model with two time-scales: the macro and micro time-scales [43]. The state-space model can be written as shown in the Eq. (11).

$$
\left\{\begin{array}{c}
\beta_{k}=\beta_{k-1}+r_{k-1} \\
X_{k, l}=f\left(X_{k, l-1}, u_{k, l-1}, \beta_{k-1}\right)+w_{k, l-1} \\
Y_{k, l}=g\left(X_{k, l}, u_{k, l-1}, \beta_{k-1}\right)+v_{k, l}
\end{array}\right.
$$

In Eq. (11), $X_{k, l}$ is the vector of system states at the time $t_{k, l}=t_{k, l-l}+\Delta t$, and $k$ and $l$ being the indices of macro and micro time-scales respectively; $u_{k, l}$ is the vector of observed exogenous inputs; $\beta_{k}$ is the vector of system model parameters at the time $t_{k, 0} ; Y_{k, l}$ is the vector of system measurements; $w_{k, l}$ and $r_{k}$ are the vectors of process noise with covariance $Q^{w}$ and $Q^{r}$ for states and model parameters, respectively; $v_{k, l}$ is the vectors of measurement noise with covariance $Q^{v} ; f(,,$,$) and g(,,$,$) are the state transition and measurement functions, respectively. L$ represents the level of time-scale separation and that $t_{k, 0}=t_{k-1, L}$.

The maximum available capacity $C_{\max }$ is selected as parameter $\beta$. Combined with the definition of SOC and the battery model, the discrete state-space equation can be obtained as shown in Eq. (12).

$$
\left\{\begin{array}{c}
C_{\max , k}=C_{\max , k-1}+r_{k-1} \\
{\left[\begin{array}{c}
S O C_{k, l} \\
U_{p, k, l}
\end{array}\right]=\left[\begin{array}{cc}
1 & 0 \\
0 & \exp (-\Delta t / \tau)
\end{array}\right]\left[\begin{array}{l}
S O C_{k, l-1} \\
U_{p, k, l-1}
\end{array}\right]+\left[\begin{array}{c}
-\eta_{\frac{\Delta t}{C_{\max }}} \\
R_{p}(1-\exp (-\Delta t / \tau))
\end{array}\right] i_{k, l-1}+w_{k, l-1}} \\
U_{L, k}=U_{O C, k}\left(S O C_{k}, T\right)-U_{p, k}-i_{k-1} R_{O}+v_{k, l}
\end{array}\right.
$$

Kalman filter is an optimized autoregressive data processing algorithm. The basic principle of the algorithm is to use the minimum mean square error as the best estimation criterion, and use the state-space model of signal and noise to introduce the relationship between state variables and observed variables by establishing state equations and observation equations model. The dual Kalman filter (DKF) method is a commonly used technique to simultaneously 
estimate the states and model parameters. The algorithm of multi-scale dual extended Kalman filter is shown in Table 2 and the detail can get in reference [43].

Table 2 Summary of multi-scale dual extended Kalman filter algorithm

1.Macro EKF for parameter estimation

a) Initialization:

$\beta_{0}=E\left[\beta_{0}\right], P_{\beta, 0}=E\left[\left(\beta_{0}-\beta_{0}\right)\left(\beta_{0}-\beta_{0}\right)^{T}\right]$

b) Iterative calculation according to formula

$\bigotimes_{k-1, L}=f_{0 \rightarrow L}\left(X_{k-1,0}, u_{k-1,0: L-1}, \bar{\beta}_{k}^{-}\right)$

$\beta_{k}^{-}=\beta_{k-1}, P_{\beta, k}^{-}=P_{\beta, k-1}+Q_{k-1}^{r}$

$K_{\beta, k}=P_{\beta, k}^{-} C_{\beta, k}^{T}\left[C_{\beta, k} P_{\beta, k}^{-} C_{\beta, k}^{T}+Q_{w, k}\right]^{-1}$

$\beta_{k}=\beta_{k}^{-}+K_{\beta, k}\left(X_{k-1, L}-X_{k-1, L}\right)$

$P_{\beta, k}=\left(I-K_{\beta, k} C_{\beta, k}\right) P_{\beta, k}^{-}$

Where

$C_{\beta, k}=\left.\frac{\mathrm{d} f_{0 \rightarrow L}\left(X_{k-1,0}, u_{k-1,0: l}, \beta\right)}{\mathrm{d} \beta}\right|_{\beta=\overline{\beta_{k}}}$
2. Micro EKF for state estimation

a) Initialization:

$$
X_{0}=E\left[X_{0}\right], P_{X, 0}=E\left[\left(X_{0}-X_{0}\right)\left(X_{0}-X_{0}\right)^{T}\right]
$$

b) Iterative calculation according to formula

$$
\begin{aligned}
& X_{k, l}^{-}=f\left(X_{k, l-1}, u_{k, l-1}, \beta_{k-1}^{-}\right), P_{k, l}^{-}=A_{k, l-1} P_{k, l-1} A_{k, l-1}^{T}+Q_{k, l-1}^{w} \\
& K_{X, k, l}=P_{k, l}^{-} C_{X, k, l}^{T}\left(C_{X, k, l} P_{k, l}^{-} C_{X, k, l}^{T}+Q_{v, k}\right) \\
& X_{k, l}=X_{k, l}^{-}+K_{X, k, l}\left[Y_{k, l}-g\left(X_{k, l}^{-}, u_{k, l-1}, \beta_{k}^{-}\right)\right] \\
& P_{k, l}=\left(I-K_{X, k, l} C_{X, k, l}^{T}\right) P_{k, l}^{-}
\end{aligned}
$$

Where

$$
\begin{gathered}
A_{k, l-1}=\left.\frac{\partial f\left(X, u_{k, l-1}, \bar{\beta}_{k}^{-}\right)}{\partial X}\right|_{X=X_{k, l-1}} \\
C_{X, k, l}=\left.\frac{\partial g\left(X, u_{k, l-1}, \beta_{k}^{-}\right)}{\partial X}\right|_{X=X_{k, l}^{-}}
\end{gathered}
$$

\subsubsection{Adaptive square root in the extended Kalman filter}

The SOC of LIB is affected by factors such as temperature, charge and discharge state, self-discharge, aging, etc. The internal chemical reaction is dynamic and its characteristics are highly nonlinear. These characteristics make SOC estimation difficult. In recent years, the Kalman filter is widely used in SOC estimation. But the realization of the covariance of the Kalman filter has numerical problems, which has been proved by many documents[35, 36]. A square root decomposition of the state covariance matrix is proposed based on the extended Kalman algorithm. On the EKF algorithm, the error variance matrix $P_{k}$ is decomposed. Make $P_{k}=S_{k} S_{k}^{T}$, instead of calculating the covariance matrix, iteratively update the covariance decomposition matrix $S_{k}$. This can ensure that the covariance matrix is non-negative definite, avoiding some algorithm divergence problems caused by the limited word length of the computer.

(1) State variables and covariance time updates as shown in Eq. (13).

$$
\left\{\begin{array}{l}
X_{k, l}=f\left(X_{k, l-1}, u_{k, l-1}, \beta_{k-1}^{-}\right) \\
S_{k, l}^{-} \stackrel{Q R}{\longleftarrow}\left[\begin{array}{l}
S_{k, l-1}^{T} A_{k, l-1}^{T} \\
\left(\left(Q_{k, l-1}^{w}\right)^{1 / 2}\right)^{T}
\end{array}\right]^{T}
\end{array}\right.
$$

(2) The corresponding Kalman gain calculation changes as shown in Eq. (14). 


$$
\left\{\begin{array}{c}
K_{X, k, l}=S_{k, l}^{-} F_{k, l} \alpha_{k, l} \\
\alpha_{k, l}=\left[F_{k, l}^{T} F_{k, l}+Q_{k, l}^{v}\right]^{-1} \\
F_{k, l}=S_{k, l-1}^{T} C_{X, k, l} \\
\gamma_{k, l}=\frac{1 \pm \sqrt{\alpha_{k, l} Q_{k, l}^{v}}}{1-\alpha_{k, l} Q_{k, l}^{v}}
\end{array}\right.
$$

(3) State variables and covariance measure updates as shown in Eq. (15).

$$
\left\{\begin{array}{l}
\tilde{y}_{k, l}=Y_{k, l}-g\left(X_{k, l}^{-}, u_{k, l-1}, \hat{\beta}_{k-1}^{-}\right) \\
X_{k, l}=X_{k, l}^{-}+K_{X, k, l} \tilde{y}_{k, l} \\
S_{k, l}=S_{k, l}^{-}\left[I-\alpha_{k, l} \gamma_{k, l} F_{k, l} F_{k, l}^{T}\right]
\end{array}\right.
$$

Since the covariance matrix $P_{\mathrm{k}}$ is decomposed, at least it is guaranteed that $P_{\mathrm{k}}$ is always non-negative definite, which can overcome the filter divergence caused by the limited word length of the computer. In Eq. (15), $I$ is identity matrix, $\tilde{y}_{k}$ is residual, and Sage-Husa adaptively updates the noise variables and by comparing the final estimated value with the estimated value. To make the estimation of noise more accurate and avoid the influence of the observed value, this paper considers the noise at the previous moment and at the specific moment of time, and adopts the weighting coefficients $d_{k, l n}$ where $d_{k, l}=(1-b) /\left(1-b^{n+1}\right), \mathrm{n}=0,1, \ldots . b$ is the forgetting factor. In practice, the smaller the value of $b$, the smaller the impact at the previous moment; if the value of $b$ is small, the estimated noise will oscillate, so it can be determined according to the specific situation. Then the calculation formula of the noise matrix is as shown in Eq. (16).

$$
\left\{\begin{array}{l}
Q_{k, l}^{w}=\left(1-d_{k, l-1}\right) Q_{k, l-1}^{w}+d_{k, l-1}\left(K_{X, k, l} \tilde{y}_{k, l} \tilde{y}_{k, l}^{T} K_{X, k, l}^{T}+P_{k, l}-A_{X, k, l} P_{k, l-1} A_{X, k, l}^{T}\right) \\
Q_{k, l}^{v}=\left(1-d_{k, l-1}\right) Q_{r, k-1}+d_{k, l-1}\left(\tilde{y}_{k, l} \tilde{y}_{k, l}^{T}-C_{X, k, l} P_{k, l}^{-} C_{X, k, l}^{T}\right)
\end{array}\right.
$$

The process noise and observation noise are corrected by Eq. (16), and the ASREKF algorithm is combined with Eq. (13) to Eq. (16) like Table 3.

Table 3 Implementation of the ASREKF algorithm.

a) Initialization:

$$
\mathbb{X}_{0}=E\left[X_{0}\right], P_{X, 0}=E\left[\left(X_{0}-X_{0}\right)\left(X_{0}-X_{0}\right)^{T}\right]=S_{0} S_{0}^{T}
$$

b) Time-update equations

$$
X_{k, l}=f\left(X_{k, l-1}, u_{k, l-1}, \beta_{k-1}^{-}\right), S_{k, l}^{-} \stackrel{Q R}{\longleftarrow}\left[\begin{array}{l}
S_{k, l-1}^{T} A_{k, l-1}^{T} \\
\left(\left(Q_{k, l-1}^{w}\right)^{1 / 2}\right)^{T}
\end{array}\right]^{T}
$$

c) Calculate the Kalman gain coefficient 


$$
\begin{aligned}
& K_{X, k, l}=S_{k, l}^{-} F_{k, l} \alpha_{k, l} \\
& \alpha_{k, l}=\left[F_{k, l}^{T} F_{k, l}+Q_{k, l}^{v}\right]^{-1} \quad \gamma_{k, l}=\frac{1 \pm \sqrt{\alpha_{k, l} Q_{k, l}^{v}}}{1-\alpha_{k, l} Q_{k, l}^{v}} \\
& F_{k, l}=S_{k, l-1}^{T} C_{X, k, l}
\end{aligned}
$$

c) Measurement-update equations

$$
\begin{aligned}
& \tilde{y}_{k}=Y_{k, l}-g\left(X_{k, l}^{-}, u_{k, l-1}, \hat{\beta}_{k-1}^{-}\right) \\
& X_{k, l}=X_{k, l}^{-}+K_{X, k, l} \tilde{y}_{k} \\
& S_{k, l}=S_{k, l}^{-}\left[I-\alpha_{k, l} \gamma_{k, l} F_{k, l} F_{k, l}^{T}\right]
\end{aligned}
$$

e) Noise covariance matrices updated iteratively

$$
\left\{\begin{array}{l}
Q_{k, l}^{w}=\left(1-d_{k, l-1}\right) Q_{k, l-1}^{w}+d_{k, l-1}\left(K_{X, k, l} \tilde{y}_{k, l} \tilde{y}_{k, l}^{T} K_{X, k, l}^{T}+P_{k, l}-A_{X, k, l} P_{k, l-1} A_{X, k, l}^{T}\right) \\
Q_{k, l}^{v}=\left(1-d_{k, l-1}\right) Q_{r, k-1}+d_{k, l-1}\left(\tilde{y}_{k, l} \tilde{y}_{k, l}^{T}-C_{X, k, l} P_{k, l}^{-} C_{X, k, l}^{T}\right)
\end{array}\right.
$$

Then, Thevenin model parameter online identification based on FFRLS, micro EKF in Table 1 replaced by ASREKF shown in Table 2. A multiscale framework with ASREKF algorithm can be obtained and the implementation flowchart is shown in Fig.2.

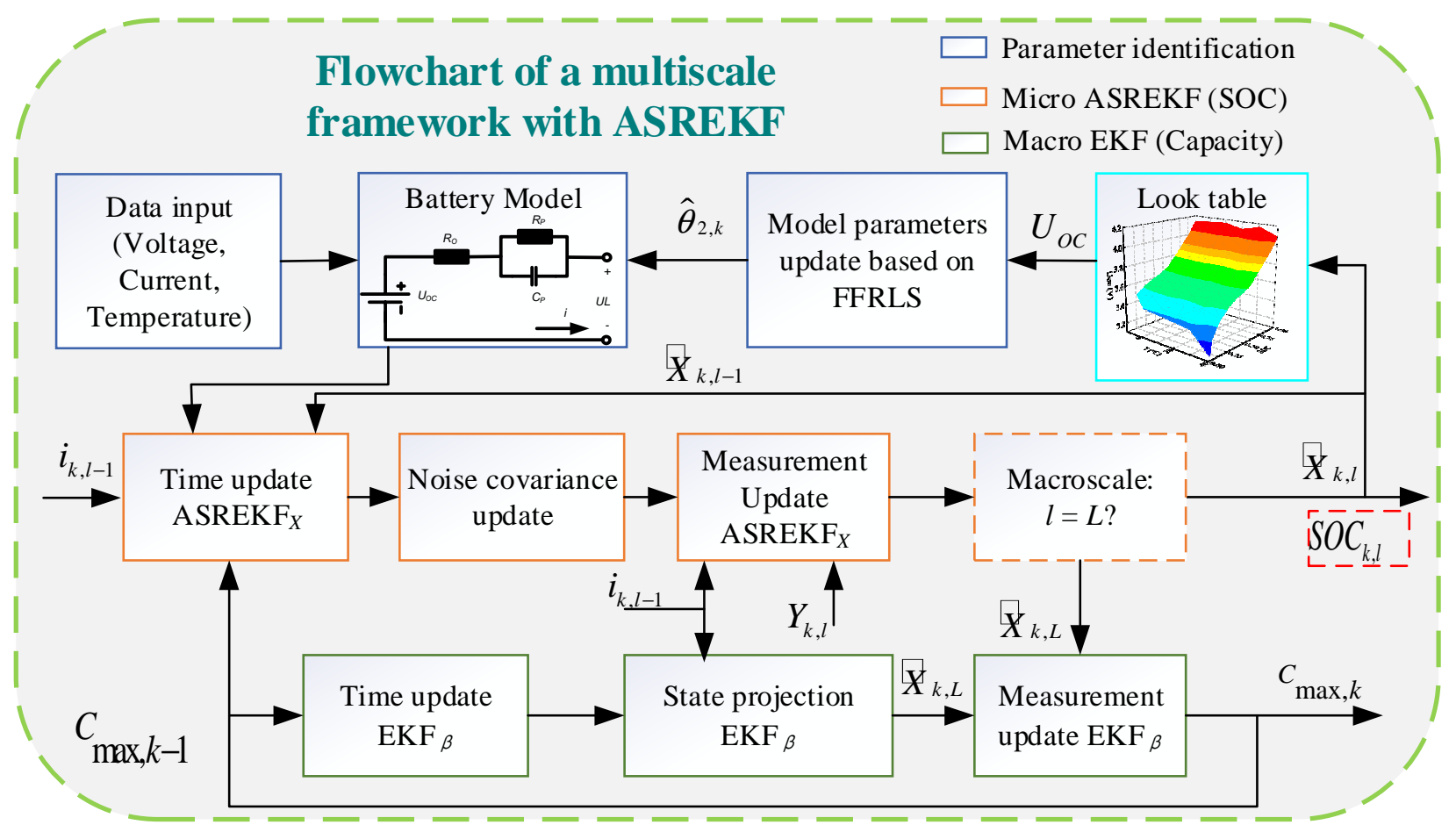

Fig. 2 Flowchart of a multiscale framework with ASREKF for battery SOC and capacity estimation.

3. Experimental analysis

\subsection{Test platform construction}

The NCM50Ah ternary power lithium battery was used as the experimental object, and its rated capacity was $50 \mathrm{Ah}$, the basic information of the battery is shown in Table 4. The battery test equipment is the Yakeyuan BTS 750-200-100-4, which has a maximum charge and discharge power of $750 \mathrm{~W}$, a maximum current of $100 \mathrm{~A}$, and a maximum voltage of $200 \mathrm{~V}$. The temperature chamber is s Bell Test Equipment Co., Ltd. BTT-331C. The entire test bench is shown in Fig. 3. 
Table.4 Basic technical parameters of the battery

\begin{tabular}{cc|cc}
\hline Cell nominal capacity/Ah & 50 & Standard charge current & $1 \mathrm{C}$ \\
Rated voltage/V & 3.65 & Standard discharge current & $3 \mathrm{C}$ \\
Charge cut-off voltage/V & $4.2 \pm 0.05$ & Maximum load current & $5 \mathrm{C}$ \\
Discharge cutoff voltage/V & $2.65 \pm 0.05$ & Internal resistance/ $\mathrm{m} \Omega$ & 0.8 \\
Size: $1 * \mathrm{w} * \mathrm{~h} / \mathrm{mm}$ & $148 \times 27 \times 93$ & Working temperature/ & $-20 \sim 60$ \\
\hline
\end{tabular}

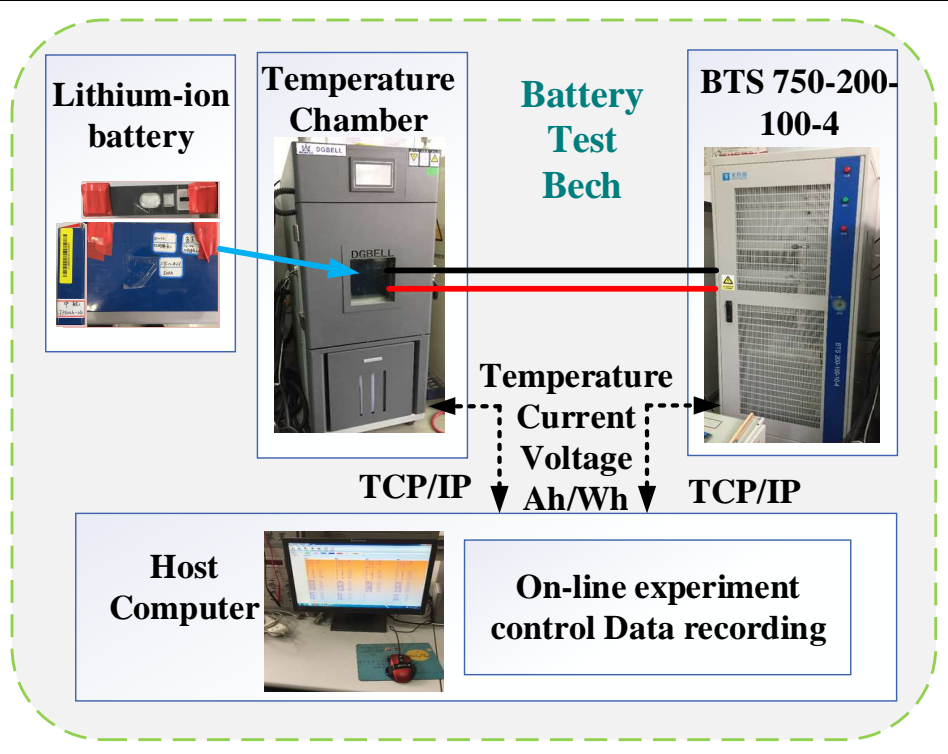

Fig. 3 Battery test bench

\subsection{Maximum available capacity at different temperatures}

The capacity measurement test at different temperatures has steps as follows: Place the LIB in the incubator, adjust the incubator to $25 \square$, and fully charge the battery with constant current and constant voltage (CC-CV). The specific steps are as follows: charge to a constant voltage when the LIB voltage is $4.2 \mathrm{~V}$ with a constant current of $50 \mathrm{~A}$ until the current is less than 2.5A. Adjust the temperature of the incubator and perform a constant current discharge test on the LIB at $1 \mathrm{C}$ (here 50A) to obtain the maximum usable capacity at this temperature. Finally, the maximum capacity of the battery at different temperatures is shown in Tab. 5 .

Table.5 Maximum available capacity at different temperatures

\begin{tabular}{cccccccc}
\hline Temperatures ( $\square$ ) & -10 & 0 & 10 & 20 & 25 & 30 & 40 \\
\hline Capacity (Ah) & 40.18 & 42.92 & 45.47 & 46.97 & 47.99 & 48.75 & 49.53 \\
\hline
\end{tabular}

Temperature has a great impact on the maximum available capacity of LIB. As the temperature decreases, the maximum available capacity of the battery decreases. The maximum available capacity of LIB at $-10{ }^{\circ} \mathrm{C}$ is only $83.7 \%$ of $\mathrm{LIB}$ at $25^{\circ} \mathrm{C}$.

\subsection{LIB test under HPPC}

LIB has the advantages of high specific energy, high single battery voltage, small self-discharge rate, long charging and discharging life and no pollution. In the energy management of lithium batteries, accurate estimation of SOC is the key to power system operation, so it is of great significance. External characteristic from the battery to the battery 
model bridge between internal states. The battery performance of state estimation, analysis and evaluation of science also play a fundamental role. The change rule of each parameter is obtained by the Hybrid Pulse Power Characteristic (HPPC) [44] experiment, in which the voltage and current change are shown in Fig. 4(a). Fig. 4(b) is the HPPC current-voltage curve made at $25^{\circ} \mathrm{C}$.

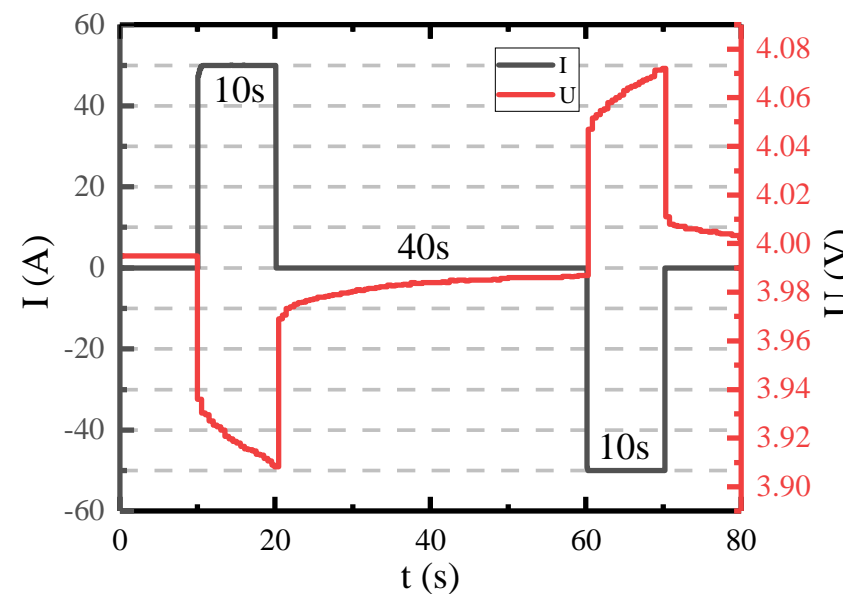

(a) Single HPPC profile $\left(\mathrm{SOC}=0.787,25^{\circ} \mathrm{C}\right)$

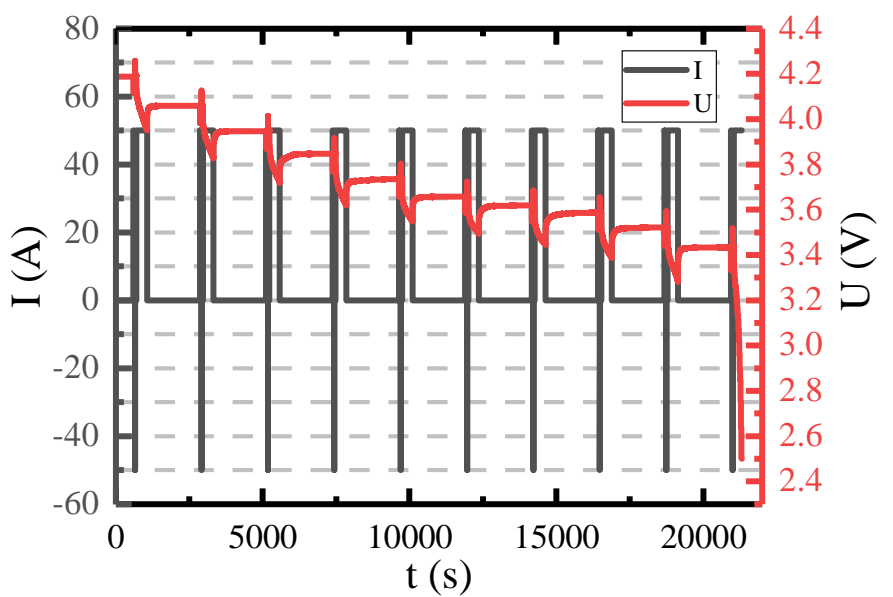

(b) Complete HPPC curve $\left(25^{\circ} \mathrm{C}\right)$

Fig. 4 HPPC experiment and results analysis

The $U_{O C}$ is determined by above HPPC experiments at different temperatures and different SOC values. After the battery is left for half an hour, there is basically no change in the terminal voltage, which can be regarded as equal to the $U_{O C}$. The results are shown in Fig. 5(a). The offline parameters identification results obtained at different SOC points according to the least-square algorithm are shown in Fig. 5(b) (c) (d).

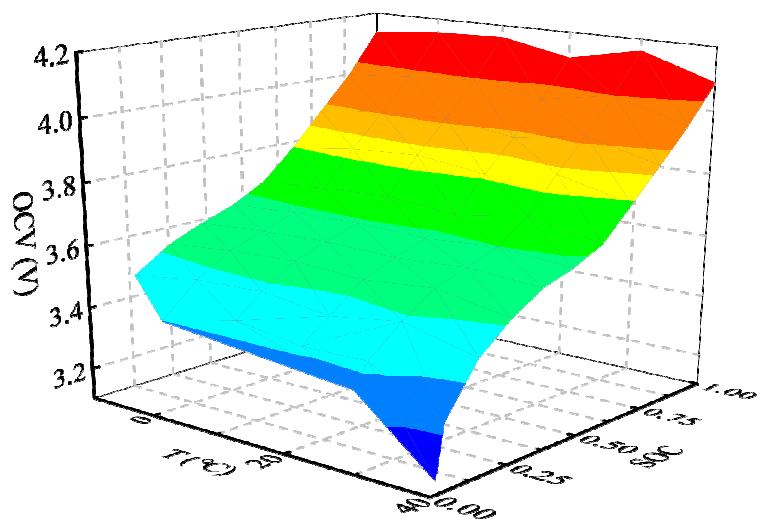

(a) Open circuit voltage $U_{O C}$

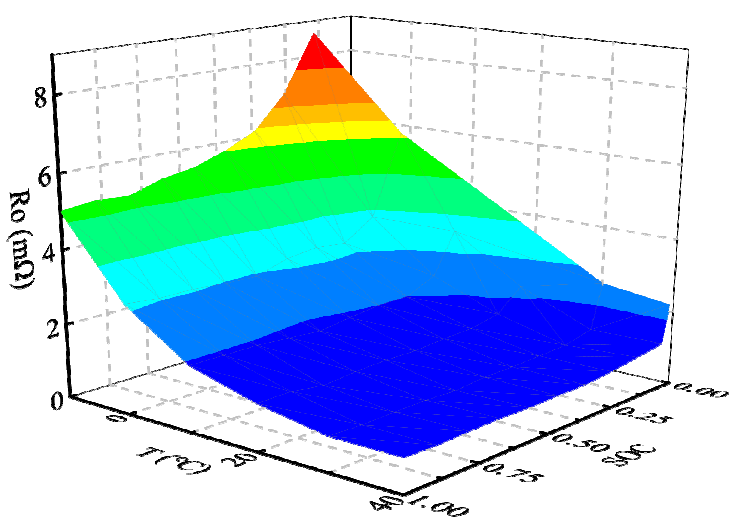

(b) Ohmic resistance $R_{O}$ 


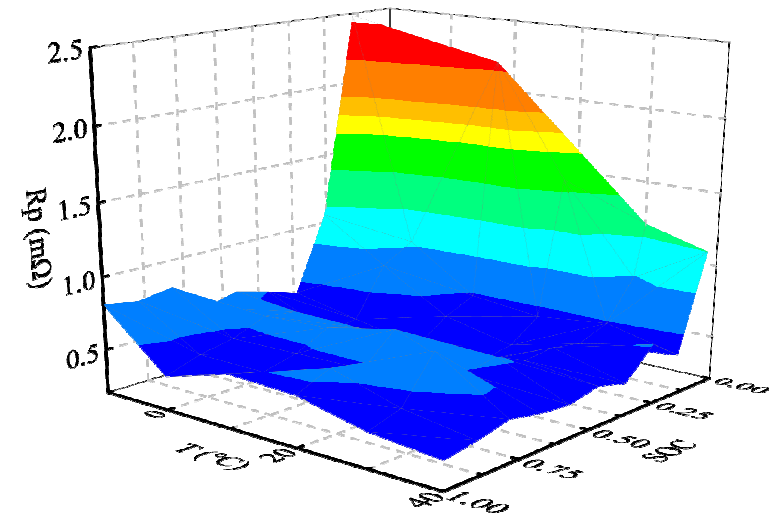

(c) Polar resistance $R_{P}$

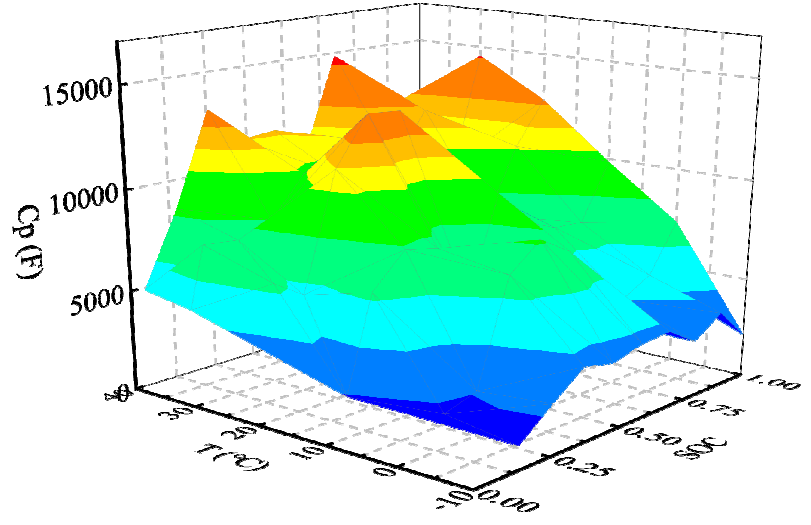

(d) Polar capacitance $C_{P}$

Fig. 5 Model parameters identification results by HPPC

In fig. 5(a), it can be seen from the figure that the $U_{O C}$ change trend at different temperatures is consistent. When the SOC is greater than 0.2, the $U_{O C}$ difference is not large at different temperatures; when the SOC is less than 0.2, the difference becomes large, and when the temperature is low, the $U_{O C}$ is high. Due to occasional error in the battery test capacity and the SOC calibration decreasing from 1 to 0 , these errors will accumulate.

In fig. 5(b), as can be seen from the figure, when the SOC is greater than 0.2 , the $R_{O}$ value is relatively smooth at each temperature without significant change and it can be taken as the $R_{O}$ does not change; when the SOC is less than 0.2 , the $R_{O}$ value rapidly increases. At the same time, the $40{ }^{\circ} \mathrm{C}$ and $30^{\circ} \mathrm{C}$ curves basically coincide, and it can be considered that the $R_{O}$ is the same. As the temperature decreases, the $R_{O}$ increases, and every $10{ }^{\circ} \mathrm{C}$ decrease, the $R_{O}$ accelerates. The effect of temperature on the internal resistance can be observed in the Fig. 5. The lower the temperature, the slower the ion transmission inside the battery and the greater the internal resistance of the battery. The charge response impedance increases more significantly with decreasing temperature.

In fig. 5(c), as can be seen from the figure, the polarization resistance $R_{P}$ at different temperatures has the same trend. When SOC is greater than 0.2 , the $R_{P}$ does not change significantly; when SOC is less than 0.2 , the $R_{P}$ becomes larger, and the $R_{P}$ at different temperatures is not clearly distinguished, indicating that the influence of temperature change on $R_{P}$ is relatively small.

In fig. 5(d), it can be seen from the figure that the change trend of the polarization capacitance $C_{P}$ at different temperatures is not obvious compared with $R_{O}$ and $R_{P}$. When SOC is less than 0.2 , the $C_{P}$ becomes smaller at different temperatures. As the temperature decreases, the $C_{P}$ will decrease slightly.

The FFRLS algorithm is a widely used online parameter identification algorithm [34, 45], The details are in section 2.3. The model parameters are estimated at the same time when estimating SOC. To verify the reliability of the model and algorithm, the results obtained based on HPPC conditions are shown in Fig. 6. The model prediction voltage and the measured voltage are compared in Fig. 6 (a) and (b) with different SOC interval. Fig. 6 (c) is the capacity estimation curve, the initial error is $2 \mathrm{Ah}$, the capacity cannot be corrected when the charge and discharge current is 0 . Fig. 6 (d) is the SOC estimation results. In Fig. 6 (d), SOC1 is reference SOC, SOC2 is estimation result by EKF, and 
SOC2 is estimation result by ASREKF. The numerical results of the algorithm performance are shown in table 7. The maximum absolute errors (MAEs) of EKF and ASREKF are 0.0226 and 0.0105 , respectively. The root mean square errors (RMSEs) of EKF and ASREKF are 0.0258 and 0.0131, respectively. As can be seen from table 7 and Fig. 6, the proposed ASREKF can achieve accurate SOC estimation.

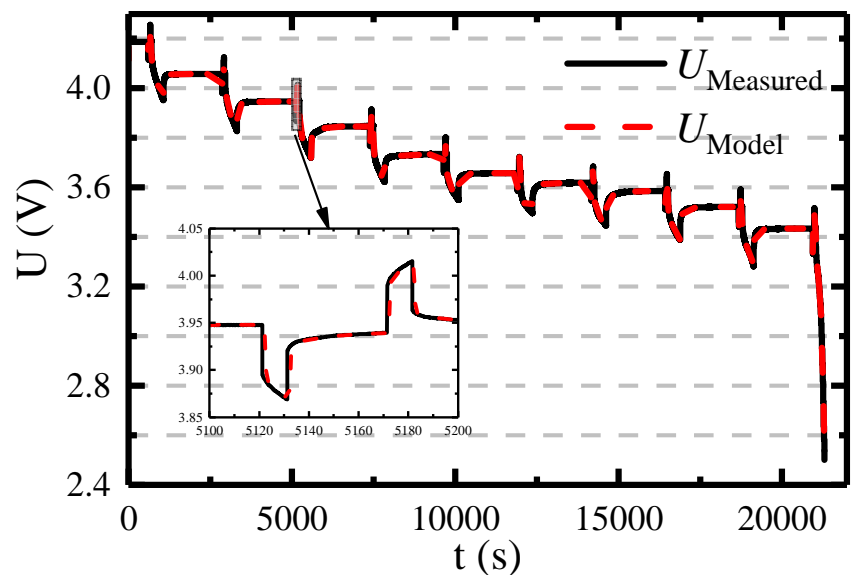

(a) Voltage profiles

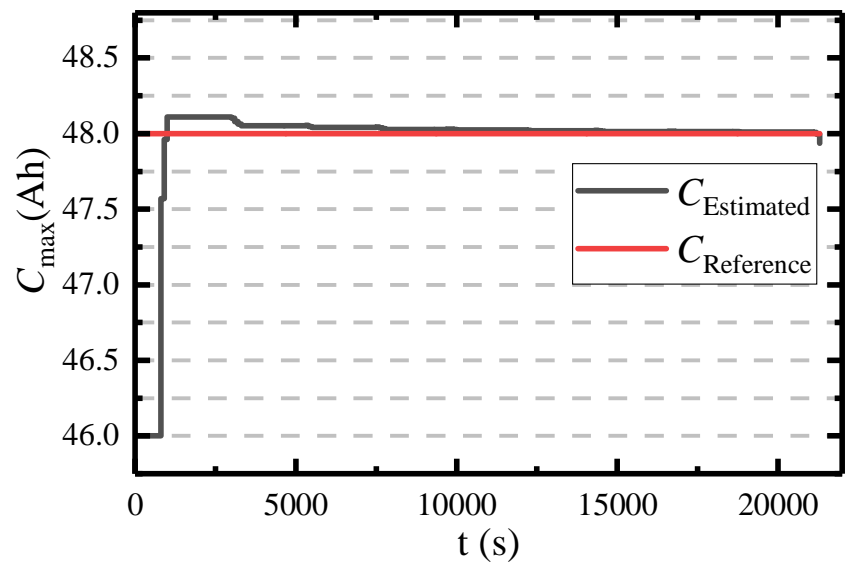

(c) Maximum usable capacity estimation

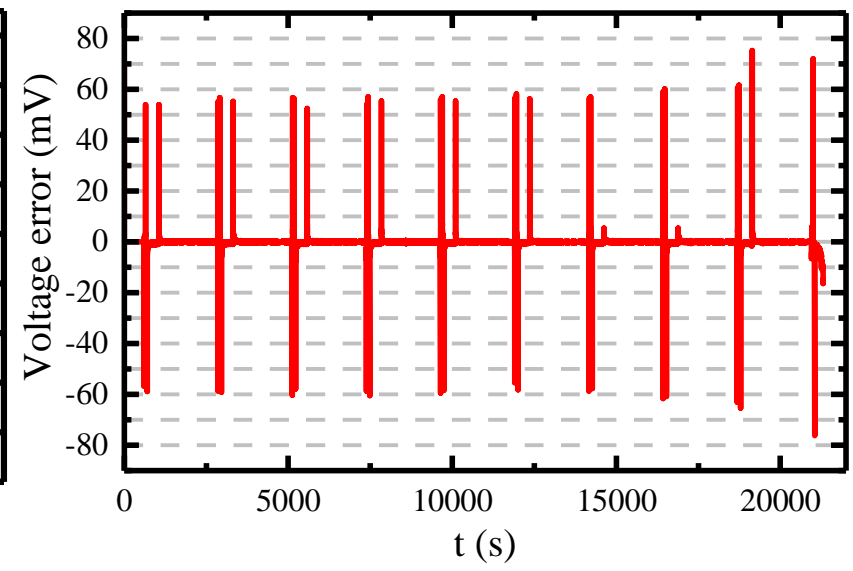

(b) Model voltage prediction error

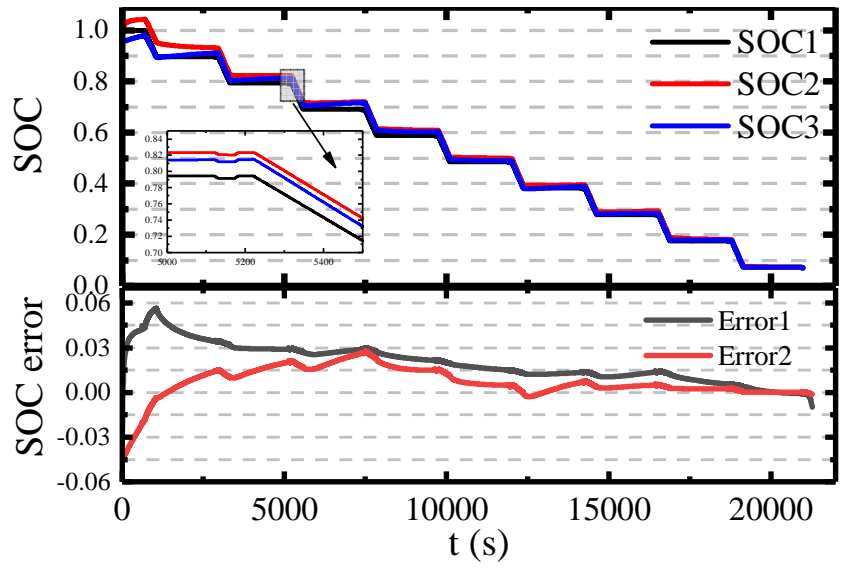

(d) SOC estimation results

Fig. 6 Algorithm verification based on HPPC

\subsection{LIB test under BBDST}

To further verify the reliability of the proposed method, more complex working conditions based on Beijing Bus Dynamic Stress Test (BBDST) are performed on the ternary LIB. The BBDST conditions which is shown in table 6 simulate the acceleration, deceleration, uniform speed, rapid acceleration, coasting, and braking of the vehicle by charging and discharging the battery with different powers and different durations.

Table.6 Beijing Bus Dynamic Stress Test

\begin{tabular}{ccccc}
\hline Step & LIB test power $/ \mathrm{w}$ & Single step time/s & total time/s & Working condition \\
\hline 1 & 75 & 21 & 21 & Start \\
2 & 145 & 12 & 33 & Accelerate \\
3 & 9 & 16 & 49 & Sliding \\
4 & -30 & 6 & 55 & Brake \\
5 & 75 & 21 & 76 & Accelerate \\
6 & 9 & 16 & 92 & Sliding \\
7 & -30 & 6 & 98 & Brake \\
\hline
\end{tabular}




\begin{tabular}{ccccc}
\hline 8 & 145 & 9 & 107 & Accelerate \\
9 & 185 & 6 & 113 & Rapid accelerate \\
10 & 75 & 21 & 134 & Accelerate \\
11 & 9 & 16 & 150 & Sliding \\
12 & -30 & 6 & 156 & Brake \\
13 & 145 & 9 & 165 & Accelerate \\
14 & 185 & 6 & 171 & Rapid accelerate \\
15 & 75 & 21 & 192 & Accelerate \\
16 & 9 & 16 & 208 & Sliding \\
17 & -70 & 9 & 217 & Brake \\
18 & -30 & 6 & 229 & Brake \\
19 & 9 & 71 & 300 & Parking \\
\hline
\end{tabular}

This test method is as follows: the LIB is charged according to the given charging mechanism, left at open-circuit state for $3 \mathrm{~h}$ after fully charged, then tested according to BBDST cycle conditions until the voltage of the battery reaches the minimum restriction. To better simulate the actual operation of the car, the experiment will be carried out once at high and low temperatures, respectively.

The experimental verification results under high temperature conditions are shown in Fig. 7. The current profiles are plotted in Fig. 7(a). The measured voltage and predicted voltage is shown in Fig. 7(b). The voltage prediction error and temperature are shown in Fig. 7(c). The SOC estimation results based on EKF and ASREKF are shown in Fig. 7(d). In Fig. 7(d), SOC1 is the reference SOC, SOC2 is the estimation results based on ASREKF, and SOC3 is the estimation results based on EKF. The numerical results of the algorithm performance are shown in table 7.

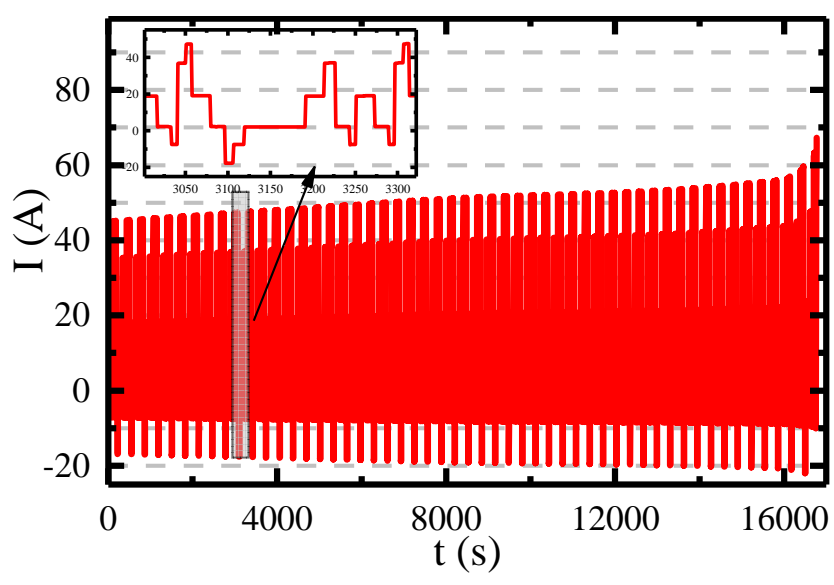

(a) BBDST cycle current profile
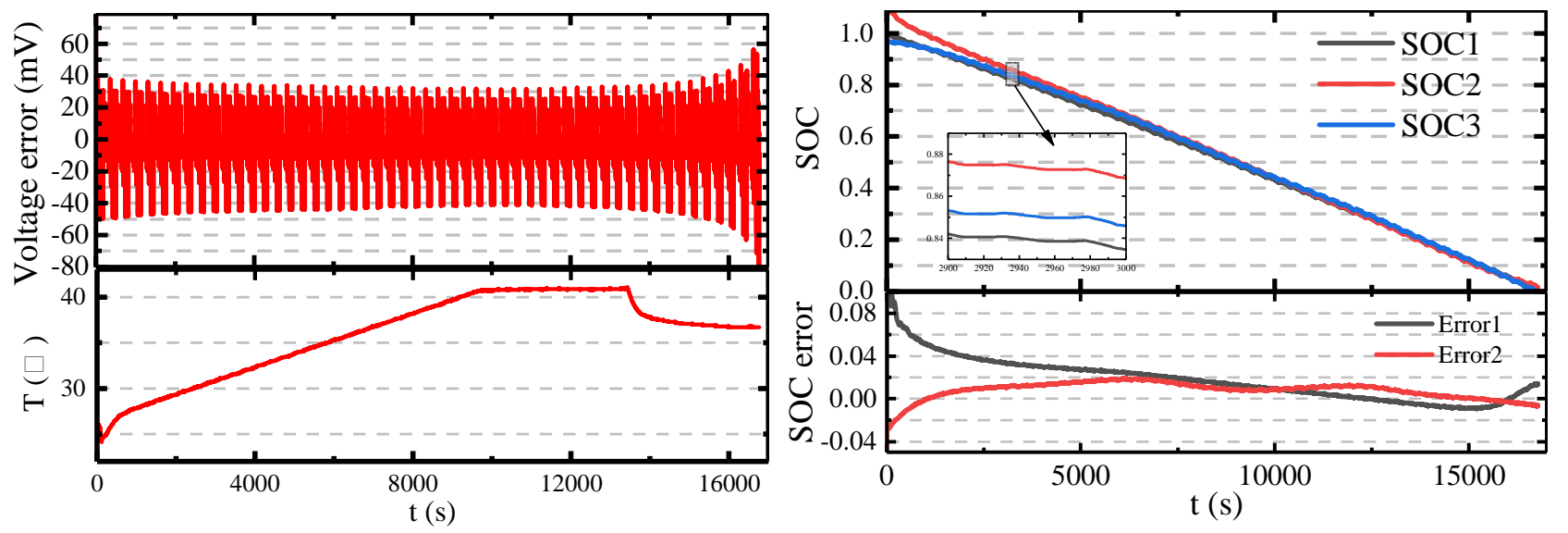
Fig. 7 SOC estimation results based on high-temperature BBDST

The experimental verification results under low temperature conditions are shown in Fig. 8. The current profiles are plotted in Fig. 8(a). The measured voltage and predicted voltage is shown in Fig. 8(b). The voltage prediction error and temperature are shown in Fig. 8(c). The SOC estimation results based on EKF and ASREKF are shown in Fig. 8(d). In Fig. 7(d), SOC1 is the reference SOC, SOC2 is the estimation results based on ASREKF, and SOC3 is the estimation results based on EKF. The numerical results of the algorithm performance are shown in table 7.

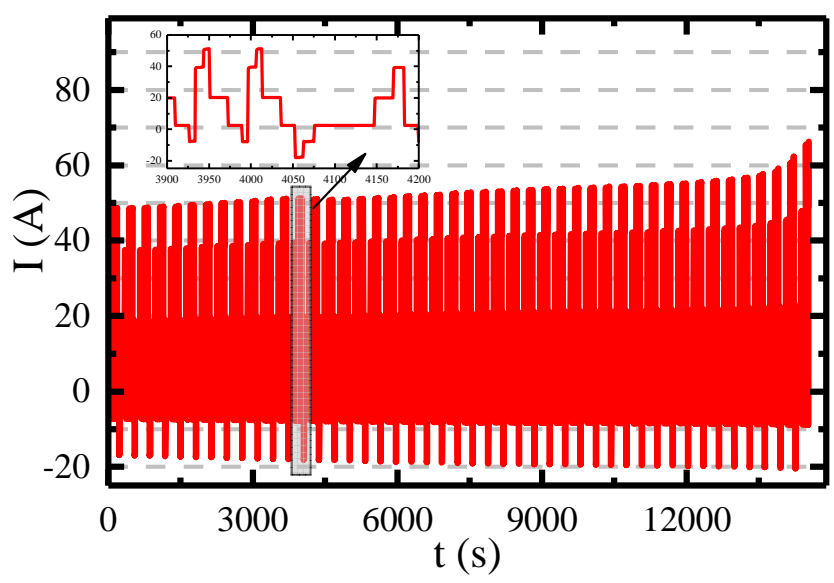

(a) BBDST measued current curve

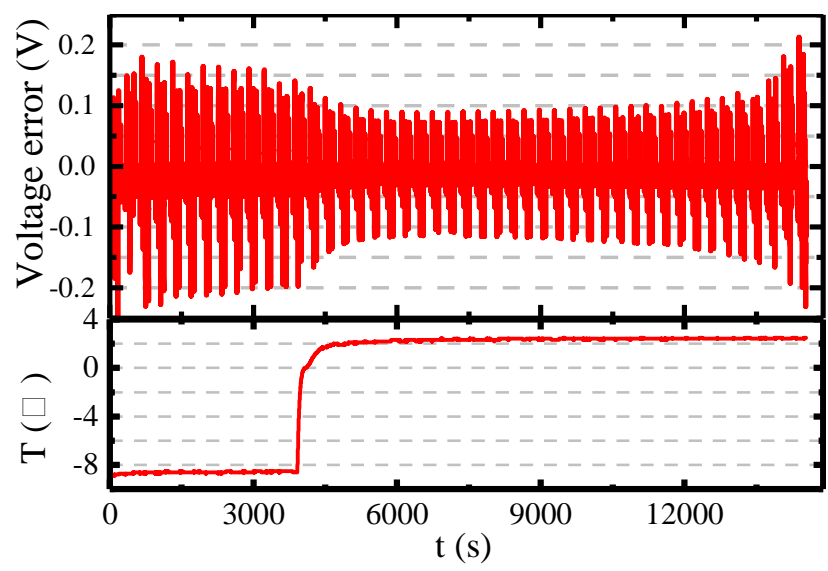

(c) BBDST voltage errror and temperature curve

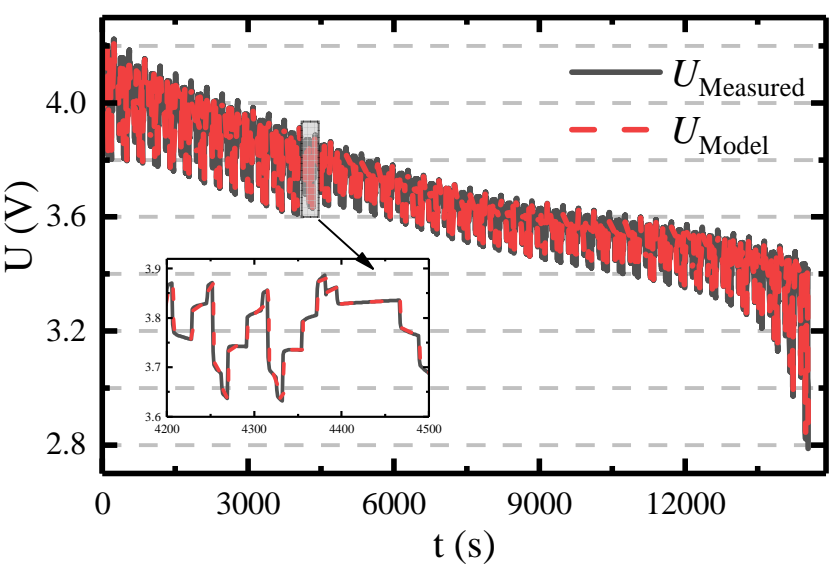

(b) BBDST measured and model voltage curve

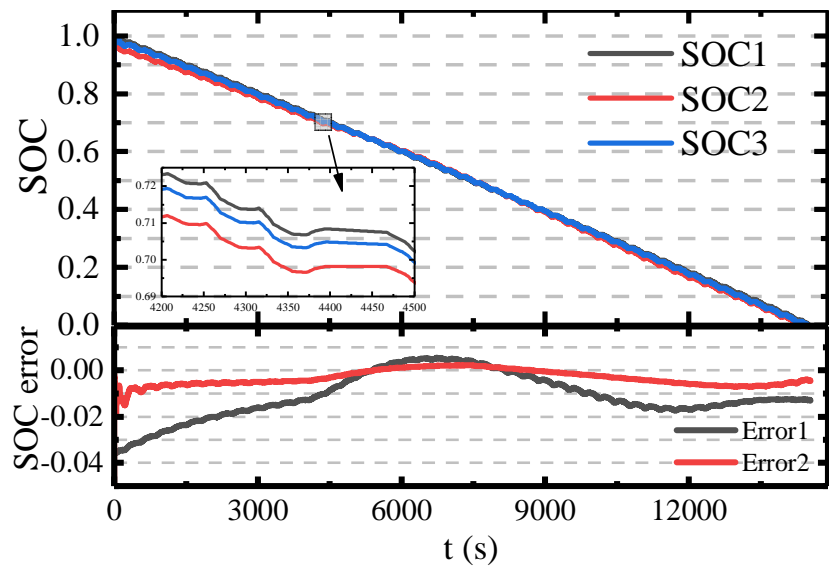

(d) SOC estimation results and error curve

Fig. 8 SOC estimation results based on low-temperature BBDST

As can be seen from Fig. 7 and Fig. 8, compared with the LIB at high temperature, the LIB voltage changes more drastically at low temperature. At the end of discharge, the voltage changes drastically at low SOC. This reason is that the model parameters change with temperature and SOC, which are consistent with those shown in Figure 5. The SOC estimation errors under different working conditions are shown in Table 7. The results under variable temperature and complex working conditions indicate that accurate estimation result can be obtained by the proposed method.

Table.7 Comparisons of MAE and RMSE

$\operatorname{HPPC}\left(25^{\circ} \mathrm{C}\right)$

BBDST (high-temperature)

BBDST (low-temperature)

$\begin{array}{lllll}\text { MAE } & \text { RMSE } & \text { MAE } & \text { RMSE }\end{array}$




\begin{tabular}{ccccccc}
\hline EKF & 0.0226 & 0.0258 & 0.0171 & 0.0246 & 0.0124 & 0.0149 \\
ASREKF & 0.0105 & 0.0131 & 0.0085 & 0.0109 & 0.0040 & 0.0048 \\
\hline
\end{tabular}

\section{Conclusions}

In this study, for the accurate SOC estimation of the power LIB based on the low error of the Thevenin model, the advantages of long-term testing and consideration of polarization effects and a battery model based on Thevenin model at different temperatures were introduced. Transient analysis of processes is considered. The forgetting factor RLS method is employed for parameter identification. Co-estimation of capacity and SOC based on a multi-scale framework of dual Kalman filters. The ASREKF algorithm effectively reduced the influence of noise and solved the problem of filter divergence that may occur due to computer processing. It has certain reference value for the rational use and distribution of power LIB. This algorithm avoids degraded or divergent filter performance. In addition, through charging and discharging experiments at different temperatures, the temperature has a great effect on the ohmic internal resistance of ternary power LIB Between $-10{ }^{\circ} \mathrm{C}$ and $40^{\circ} \mathrm{C}$. To verify and evaluate the proposed algorithm, dynamic temperature BBDST are performed on the NCA battery. The experimental results are accurate, the experimental steps are simple and convenient.

\section{Acknowledgments}

The research was supported by National Natural Science Foundation of China (No. 61801407).

\section{References}

[1] Kumar R., Sahoo S., Joanni E., Singh R.K., Tan W.K., Kar K.K., et al. Recent progress in the synthesis of graphene and derived materials for next generation electrodes of high performance lithium ion batteries. Prog. Energy Combust. Sci. 75 (2019) 56.

[2] Kumar R., Sahoo S., Joanni E., Singh R.K., Yadav R.M., Verma R.K., et al. A review on synthesis of graphene, h-BN and MoS2 for energy storage applications: Recent progress and perspectives. Nano Res. 12 (2019) 2655-94.

[3] Graditi G., Ippolito M.G., Telaretti E., Zizzo G. Technical and economical assessment of distributed electrochemical storages for load shifting applications: An Italian case study. Renew. Sust. Energ. Rev. 57 (2016) 515-23.

[4] Zhu X.H., Macia L.F., Jaguemont J., de Hoog J., Nikolian A., Omar N., et al. Electrochemical impedance study of commercial LiNi0.80Co0.15Al0.05O2 electrodes as a function of state of charge and aging. 
Electrochimica Acta. 287 (2018) 10-20.

[5] Zheng Y.J., Ouyang M.G., Han X.B., Lu L.G., Li J.Q. Investigating the error sources of the online state of charge estimation methods for lithium-ion batteries in electric vehicles. J Power Sources. 377 (2018) 161-88.

[6] Hosseinimehr T., Ghosh A., Shahnia F. Cooperative control of battery energy storage systems in microgrids. International Journal of Electrical Power \& Energy Systems. 87 (2017) 109-20.

[7] Lim K., Bastawrous H.A., Duong V.H., See K.W., Zhang P., Dou S.X. Fading Kalman filter-based real-time state of charge estimation in LiFePO4 battery-powered electric vehicles. Appl Energ. 169 (2016) 40-8.

[8] Partovibakhsh M., Liu G.J. An Adaptive Unscented Kalman Filtering Approach for Online Estimation of Model Parameters and State-of-Charge of Lithium-Ion Batteries for Autonomous Mobile Robots. leee Transactions on Control Systems Technology. 23 (2015) 357-63.

[9] Xiong R., Cao J., Yu Q., He H., Sun F. Critical Review on the Battery State of Charge Estimation Methods for Electric Vehicles. IEEE Access. 6 (2018) 1832-43.

[10] Pan H., Lü Z., Lin W., Li J., Chen L. State of charge estimation of lithium-ion batteries using a grey extended Kalman filter and a novel open-circuit voltage model. Energy. 138 (2017) 764-75.

[11] He J.T., Feng D.W., Hu C., Wei Z.B., Yan F.J. Two-layer online state-of-charge estimation of lithium-ion battery with current sensor bias correction. International Journal of Energy Research. 43 (2019) 3837-52.

[12] Ahmad A.B., Ooi C.A., Ishak D., Teh J. State-of-Charge Balancing Control for ON/OFF-Line Internal Cells Using Hybrid Modular Multi-Level Converter and Parallel Modular Dual L-Bridge in a Grid-Scale Battery Energy Storage System. leee Access. 7 (2019) 131-47.

[13] Hoang K.D., Lee H.H. Accurate Power Sharing With Balanced Battery State of Charge in Distributed DC Microgrid. leee Transactions on Industrial Electronics. 66 (2019) 1883-93.

[14] Liu D.T., Li L., Song Y.C., Wu L.F., Peng Y. Hybrid state of charge estimation for lithium-ion battery under dynamic operating conditions. International Journal of Electrical Power \& Energy Systems. 110 (2019) 
48-61.

[15] leee Transactions on Industrial ElectronicsZheng L., Zhu J., Wang G., Lu D.D.-C., He T. Differential voltage analysis based state of charge estimation methods for lithium-ion batteries using extended Kalman filter and particle filter. Energy. 158 (2018) 1028-37.

[16] Dang X., Yan L., Xu K., Wu X., Jiang H., Sun H. Open-Circuit Voltage-Based State of Charge Estimation of Lithium-ion Battery Using Dual Neural Network Fusion Battery Model. Electrochimica Acta. 188 (2016) $356-66$.

[17] Lipu M.S.H., Hannan M.A., Hussain A., Saad M.H.M. Optimal BP neural network algorithm for state of charge estimation of lithium-ion battery using PSO with PCA feature selection. Journal of Renewable and Sustainable Energy. 9 (2017).

[18] Tian Y., Lai R., Li X., Xiang L., Tian J. A combined method for state-of-charge estimation for lithium-ion batteries using a long short-term memory network and an adaptive cubature Kalman filter. Applied Energy. 265 (2020).

[19] Jiao M., Wang D., Qiu J. A GRU-RNN based momentum optimized algorithm for SOC estimation. Journal of Power Sources. 459 (2020).

[20] Wang Y.Z., Ni Y.L., Li N., Lu S., Zhang S.D., Feng Z.B., et al. A method based on improved ant lion optimization and support vector regression for remaining useful life estimation of lithium-ion batteries. Energy Sci Eng. (2019).

[21] Zeng Z., Tian J., Li D., Tian Y. An Online State of Charge Estimation Algorithm for Lithium-Ion Batteries Using an Improved Adaptive Cubature Kalman Filter. Energies. 11 (2018) 59.

[22] Wang S.L., Shi J.Y., Fernandez C., Zou C.Y., Bai D.K., Li J.C. An improved packing equivalent circuit modeling method with the cell-to-cell consistency state evaluation of the internal connected lithium-ion batteries. Energy Sci Eng. 7 (2019) 546-56. 
[23] Xia B., Zhao X., de Callafon R., Garnier H., Nguyen T., Mi C. Accurate Lithium-ion battery parameter estimation with continuous-time system identification methods. Applied Energy. 179 (2016) 426-36.

[24] Linghu J.Q., Kang L.Y., Liu M., Hu B.H., Wang Z.F. An Improved Model Equation Based on a Gaussian Function Trinomial for State of Charge Estimation of Lithium-ion Batteries. Energies. 12 (2019).

[25] Wei Z., Zhao J., Ji D., Tseng K.J. A multi-timescale estimator for battery state of charge and capacity dual estimation based on an online identified model. Applied Energy. 204 (2017) 1264-74.

[26] Mu H., Xiong R., Zheng H., Chang Y., Chen Z. A novel fractional order model based state-of-charge estimation method for lithium-ion battery. Applied Energy. 207 (2017) 384-93.

[27] Chen Z.H., Sun H., Dong G.Z., Wei J.W., Wu J. Particle filter-based state-of-charge estimation and remaining-dischargeable-time prediction method for lithium-ion batteries. J Power Sources. 414 (2019) $158-66$.

[28] Chen C., Xiong R., Shen W.X. A Lithium-Ion Battery-in-the-Loop Approach to Test and Validate Multiscale Dual H Infinity Filters for State-of-Charge and Capacity Estimation. leee Transactions on Power Electronics. 33 (2018) 332-42.

[29] Hou J., Yang Y., He H., Gao T. Adaptive Dual Extended Kalman Filter Based on Variational Bayesian Approximation for Joint Estimation of Lithium-Ion Battery State of Charge and Model Parameters. Applied Sciences-Basel. 9 (2019).

[30] Wang S.L., Yu C.M., Fernandez C., Chen M.J., Li G.L., Liu X.H. Adaptive State-of-Charge Estimation Method for an Aeronautical Lithium-ion Battery Pack Based on a Reduced Particle-unscented Kalman Filter. J. Power Electron. 18 (2018) 1127-39.

[31] Ye M., Guo H., Cao B. A model-based adaptive state of charge estimator for a lithium-ion battery using an improved adaptive particle filter. Applied Energy. 190 (2017) 740-8.

[32] Zhu Q., Xu M.G., Liu W.Q., Zheng M.Q. A state of charge estimation method for lithium-ion batteries based 
on fractional order adaptive extended kalman filter. Energy. 187 (2019) 11.

[33] Lai X., He L., Wang S., Zhou L., Zhang Y., Sun T., et al. Co-estimation of state of charge and state of power for lithium-ion batteries based on fractional variable-order model. Journal of Cleaner Production. 255 (2020).

[34] Wang Y., Zhang C., Chen Z. An adaptive remaining energy prediction approach for lithium-ion batteries in electric vehicles. Journal of Power Sources. 305 (2016) 80-8.

[35] Kadiyala P.G., Bryson A.E., Schmidt S.F. Discrete square root filtering: A survey of current techniques. IEEE Transactions on Automatic Control. 16 (1971) 727-35.

[36] C.J. B.G.J.T. Numerical comparison of Kalman filter algorithms: Obirt determination case study. Automtica. 13 (1977) 23-35.

[37] Wang Y., Liu C., Pan R., Chen Z. Modeling and state-of-charge prediction of lithium-ion battery and ultracapacitor hybrids with a co-estimator. Energy. 121 (2017) 739-50.

[38] Zhang W.J., Wang L.Y., Wang L.F., Liao C.L. An improved adaptive estimator for state-of-charge estimation of lithium-ion batteries. Journal of Power Sources. 402 (2018) 422-33.

[39] Zou C., Klintberg A., Wei Z., Fridholm B., Wik T., Egardt B. Power capability prediction for lithium-ion batteries using economic nonlinear model predictive control. Journal of Power Sources. 396 (2018) 580-9.

[40] Wang S.L., Fernandez C., Shang L.P., Li Z.F., Yuan H.F. An integrated online adaptive state of charge estimation approach of high-power lithium-ion battery packs. Trans. Inst. Meas. Control. 40 (2018) $1892-910$.

[41] Lai X., Zheng Y., Sun T. A comparative study of different equivalent circuit models for estimating state-of-charge of lithium-ion batteries. Electrochimica Acta. 259 (2018) 566-77.

[42] Wei Z., Tseng K.J., Wai N., Lim T.M., Skyllas-Kazacos M. Adaptive estimation of state of charge and capacity with online identified battery model for vanadium redox flow battery. Journal of Power Sources. 332 (2016) 389-98. 
[43] Hu C., Youn B.D., Chung J. A multiscale framework with extended Kalman filter for lithium-ion battery SOC and capacity estimation. Applied Energy. 92 (2012) 694-704.

[44] FreedomCAR Battery Test Manual for Power-Assist Hybrid Electric Vehicles, U.S. Dept. Energy, Idaho, ID, USA. (Oct. 2003).

[45] Shen P., Ouyang M., Lu L., Li J., Feng X. The Co-estimation of State of Charge, State of Health, and State of Function for Lithium-Ion Batteries in Electric Vehicles. IEEE Transactions on Vehicular Technology. 67 (2018) 92-103. 


\section{Research highlights}

- An novel adaptive square root extended Kalman filter is proposed.

- Co-estimate capacity and state-of-charge based on multi-scale dual Kalman filter.

- The recursive least square method is employed for parameters online identification.

- The changes of lithium-ion battery model parameters with temperature are studied.

- The algorithm is validated with dynamic temperature working conditions. 